\title{
Emergence of male-biased genes on the chicken Z-chromosome: Sex-chromosome contrasts between male and female heterogametic systems
}

\author{
Hans Ellegren ${ }^{1}$ \\ Department of Evolutionary Biology, Evolutionary Biology Centre, Uppsala University, Norbyvägen 18D, SE-752 36 \\ Uppsala, Sweden
}

\begin{abstract}
There has been extensive traffic of male-biased genes out of the mammalian and Drosophila X-chromosomes, and there are also reports of an under-representation of male-biased genes on the $X$. This may reflect an adaptive process driven by natural selection where an autosomal location of male-biased genes is favored since male genes are only exposed to selection one-third of the time when $\mathrm{X}$-linked. However, there are several alternative explanations to "out-of-the-X" gene movement, including mutational bias and a means for X-linked genes to escape meiotic sex chromosome inactivation (MSCl) during spermatogenesis. As a critical test of the hypothesis that genomic relocation of sex-biased genes is an adaptive process, I examined the emergence, and loss, of genes on the chicken Z-chromosome, i.e., a female heterogametic system (males ZZ, females ZW). Here, the analogous prediction would be an emergence of male-biased genes onto, not a loss from, the Z-chromosome because $Z$ is found more often in males than autosomes are. I found that genes expressed in testis but not in ovary are highly over-represented among genes that have emerged on the Z-chromosome during avian evolution. Moreover, genes with male-biased expression are similarly over-represented among new Z-chromosomal genes. Interestingly, genes with female-biased expression have more often moved from than to the Z-chromosome. These observations show that male and female heterogametic organisms display opposing directionalities in the emergence and loss of sex-biased genes on sex chromosomes. This is consistent with theoretical models on the evolution of sexually antagonistic genes in which new mutations are at least partly dominant.
\end{abstract}

[Supplemental material is available for this article.]

Selection shapes genome evolution at the DNA sequence level, mainly manifested in changes in the primary structure of genes and other functional elements. A long-standing question in evolutionary biology is whether the organization of genes, their location in different regions of the genome, is also subject to selection (Hurst et al. 2004). A candidate case would be the genomic organization of sexually antagonistic genes, i.e., genes beneficial to one sex but either detrimental or neutral to the other (Rice 1992; Rice and Chippindale 2001; van Doorn 2009). Theoretical arguments imply that such genes are only expected to occur if the benefits accrued to one sex outweigh the disadvantages imposed to the other. However, there is an important exception to this prediction that relates to the special mode of inheritance and organization of sex chromosomes, and to cost-benefit scenarios associated with sex-linkage. For example, as far as partially or fully dominant mutations are considered, theory predicts that it should be easier for an antagonistic gene favorable to one sex to evolve on a sex chromosome that is more often present in that sex than in the other, since net selection will then be positive (Rice 1984; Charlesworth et al. 1987). This leads to the expectation that female-beneficial genes should be overrepresented, and male-beneficial genes under-represented, on the $\mathrm{X}$-chromosome in male-heterogametic organisms.

In practice, it is difficult on a large scale to determine whether genes are or have been sexually antagonistic. Several investigators have therefore used sex-biased gene expression as a proxy for sexual

\footnotetext{
${ }^{1}$ Corresponding author.

E-mail Hans.Ellegren@ebc.uu.se.

Article published online before print. Article, supplemental material, and publication date are at http://www.genome.org/cgi/doi/10.1101/gr.119065.110.
}

antagonism (see Ellegren and Parsch 2007); sex-biased gene expression may potentially represent the resolution of past sexual antagonism (e.g., Mank and Ellegren 2009). This line of thinking has been integrated with several observations of a non-random genomic distribution of genes with sex-biased expression. In Drosophila, malebiased genes are under-represented on the X (Parisi et al. 2003; Sturgill et al. 2007; Vicoso and Charlesworth 2009). If this is translated into an under-representation of male-beneficial genes on $\mathrm{X}$, it would be consistent with theory for partly or fully dominant mutations (Rice 1984). In Caenorhabditis elegans, sperm-enriched genes are nearly absent from the X-chromosome (Reinke et al. 2000). In mammals, the picture is more complex. Female-biased genes expressed in ovary and placenta are over-represented on the mouse X-chromosome (Khil et al. 2004), also as expected for dominant mutations. However, male-biased genes expressed in early stages of spermatogenesis are in excess on the mouse $\mathrm{X}$ as well (Khil et al. 2004), although genes expressed later during male meiosis are, in fact, under-represented on the X (Khil et al. 2004). Several alternative explanations to the non-random distribution of sex-biased genes have therefore been suggested (Rogers et al. 2003; Meisel et al. 2009), including escape from meiotic sex chromosome inactivation (MSCI) by genes needed to be expressed during spermatogenesis (Hense et al. 2007; Potrzebowski et al. 2008; Vibranovski et al. 2009a).

A limitation in the abovementioned empirical studies is that they survey the overall present-day gene content of different chromosomal categories rather than specifically focusing on the characteristics of those new genes that evolved in different regions of the genome (cf. Zhang et al. 2010). However, the arguments for the probability of evolution of sexually antagonistic alleles in different chromosomal categories can also be applied to different scenarios of 
movement of genes between sex chromosomes and autosomes (Moyle et al. 2010). Relevant in this context are empirical data on traffic of retrogenes between autosomes and the X-chromosome in mammals and Drosophila (Betran et al. 2002; Emerson et al. 2004; Vibranovski et al. 2009b), in particular, a pattern that has been referred to as "out-of-X movement." It has been noted that many retrotransposed gene copies moving from the X-chromosome to autosomes have acquired sex-biased gene expression and are mainly expressed in testis (Bradley et al. 2004). In the seminal study by Emerson et al. (2004), it was hypothesized that natural selection has favored the preservation of genes with male-biased function that move from the X-chromosome to autosomes: "One model of sexual antagonism (Wu and Yujun $\mathrm{Xu}$ 2003) predicts that gene copies that benefit males at a cost to females would be more likely found on the autosomes than on the X-chromosome." Note again that sex-biased gene expression is taken as a proxy for sexual antagonism.

A potentially interesting contrast in testing an adaptive scenario for the genomic distribution of new sex-biased genes is to study organisms with female heterogamety (males ZZ, females $\mathrm{ZW}$ ). In this case, male-beneficial genes in which new mutations are at least partly dominant may be expected to accumulate on the $\mathrm{Z}$-chromosome, rather than on autosomes, because $\mathrm{Z}$ is two-thirds of the time in males. With the same argument, female-beneficial genes would be expected to become less abundant on the Z-chromosome. These opposing predictions compared to systems of male heterogamety are helpful for the general argument because they allow distinguishing the expected effects of heterogamety from any other difference that pertain to autosomes and sex chromosomes. Female heterogamety is found in a diverse range of organisms. All birds are female heterogametic, and the same applies to all butterflies. A ZW sex chromosome system is also present in individual lineages of reptiles, amphibians, fishes, and crustaceans. Although draft genome sequences of a few female heterogametic bird species (chicken, Gallus gallus [International Chicken Genome Sequencing Consortium 2004]; zebra finch, Taeniopygia guttata [Warren et al. 2010]; and turkey [Dalloul et al. 2010]) and one moth (silkworm, Bombyx mori [Mita et al. 2004; Xia et al. 2004]) have been reported, the coverage of the Z-chromosome has generally not been sufficient for a comprehensive analysis of gene content. However, the recent sequencing to near completion of the chicken Z-chromosome (Bellott et al. 2010) provides a framework for a test of the emergence of new genes on sex chromosomes in a female heterogametic system. The chicken Z-chromosome is $80 \mathrm{Mb}$ in size and contains about 1000 genes. The female-specific W-chromosome is less well characterized, but it is considerably smaller, mostly non-recombining, and contains a limited number of paralogs (gametologs) to Z-linked genes as well as some female-specific genes (Ellegren 2000; International Chicken Genome Sequencing Consortium 2004). The avian ZW and the mammalian XY sex chromosomes evolved independently from different pairs of autosomes present in a vertebrate ancestor (Fridolfsson et al. 1998; Nanda et al. 1999; Bellott et al. 2010). They thereby offer parallel biological replicates to the study of sex chromosome evolution and the associated question of genome organization potentially driven by selectively favorable gene acquisitions.

\section{Results}

Bellot et al. (2010) identified 76 single-copy, or in a few cases duplicate, genes on the chicken Z-chromosome that have evolved subsequent to the emergence of avian sex chromosomes. These genes represent a mix of different evolutionary histories. Thirtyeight are novel, bird-specific genes. Twenty-five genes are located in regions of the human genome that are not syntenic with the chicken Z-chromosome and are therefore interpreted to have moved to the Z-chromosome from an ancestral autosomal location. Another nine are duplications/transpositions of autosomal bird genes where the new copy has become inserted on the Z-chromosome. The remaining four genes are not annotated. All genes contain introns and thus do not represent cases of retrotransposition.

Expression data in adult chicken gonads for 42 (Supplemental Table 1) of these 76 new genes show that $78.6 \%$ are expressed in testis, which is a significantly higher proportion than for autosomal genes $\left(61.2 \%, \chi^{2}=5.36, P=0.020\right)$ but not for other Z-linked genes in chicken $\left(68.8 \%, \chi^{2}=1.82, P=0.178\right)$. Moreover, 13 out of the 42 new $Z$ genes (31.0\%) are expressed in testis but not in ovary, significantly more than the frequency of genes showing this pattern in the whole chicken genome $\left(9.1 \%, \chi^{2}=24.35, P=\right.$ $0.0000008)$ as well as on the Z-chromosome $\left(12.1 \%, \chi^{2}=13.32, P=\right.$ 0.00026). In addition, another 11 genes expressed in both testis and ovary show male-biased expression. Thus, more than half ( 24 out of 42) of all genes recruited to the avian Z-chromosome show an association with male reproduction (Fig. 1). In contrast, only one of the recruited genes is expressed in ovary but not in testis, and only two further show female-biased expression, giving a highly significant difference between the incidences of genes associated with male and female reproduction among the new $\mathrm{Z}$ genes $(P=$ 0.000001; Fisher's exact test).

Twenty-one chicken genes have left the Z-chromosome for relocation to an autosome; expression data from adult gonads are available for 17 of these. Although the number is low, there is an interesting difference between the genes recruited to $Z$ and the "out-of-the-Z" genes: The incidence of genes with female-biased expression is significantly higher among the latter (29.4\%) than the former $(4.8 \% ; P=0.0106$; Fisher's exact test). This indicates that there has been a loss, rather than an accumulation, of female-biased genes from the avian Z-chromosome.

I also examined sex-biased expression in chicken heart and brain, i.e., two somatic tissues, for genes that have emerged on or left the Z-chromosome. Expression data from these tissues are available for 23 and 30 out of the 76 new Z-linked genes, respectively (33 genes in total). None of the genes show male-biased expression. However, overall, the number of genes on the Z-chromosome meeting the criteria for male-biased expression in somatic tissues is extremely limited (two out of 693 Z-linked genes expressed in heart and none out of $758 \mathrm{Z}$-linked genes expression in brain), reflecting the much lower proportion of sex-biased genes in somatic compared to gonadal tissue of chicken coupled with the stringent criteria necessary for defining male-biased expression of sex-linked genes given incomplete dosage compensation (Ellegren et al. 2007).

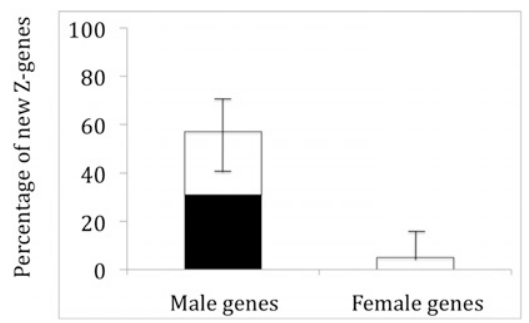

Figure 1. The percentage of male and female reproductive genes among those that have emerged on the Z-chromosome during avian evolution. Solid bars are genes specific to either testis or ovary, and open bars are genes with sex-biased expression in gonads. Error bars indicate the $95 \%$ confidence interval for the total proportion of sex-specific and sex-biased genes. 
When it comes to the 21 genes that have left the Z-chromosome for an autosomal location, there are expression data available for 11 and 12 genes from brain and heart, respectively. Among these, there is only one gene with female-biased expression in one of the tissues (brain). For all autosomal genes expressed in brain, $\approx 5 \%$ show female-biased expression (693 out of 13,750).

\section{Discussion}

These observations provide evidence that male and female heterogametic organisms display opposing directionalities when it comes to sex chromosomes as the "sink" or "source" for the evolution of new sex-biased genes. While testis-specific expression is seen among retrogenes that have left the mammalian X-chromosome (Betran et al. 2002; Emerson et al. 2004), male-biased genes have entered the avian Z-chromosome. In addition, in birds, female-biased genes tend to leave $\mathrm{Z}$, while there is some evidence for female-biased genes to be over-represented among new genes recruited to the mammalian X-chromosome (Zhang et al. 2010). This can potentially be explained by an adaptive location of genes related to the special evolutionary forces operating on sex chromosomes. Specifically, the combined data from male (Betran et al. 2002; Emerson et al. 2004; Vibranovski et al. 2009b) and female heterogametic (this study) organisms suggest that male-biased genes will preferentially move to, or evolve in, the chromosomal category that maximizes their exposure: autosomes in XY species and sex chromosomes in $\mathrm{ZW}$ species. Interestingly, analogous theoretical arguments for genes involved in female reproduction lead to the prediction that transfer of female-biased genes from sex chromosomes to autosomes should be favorable in female heterogametic organisms given that the Z-chromosome is only onethird of the time in females. The higher incidence of female-biased genes seen among genes that have left the Z-chromosome than among those that have evolved on the Z-chromosome is in accordance with this prediction.

The observation of the emergence of new male-biased genes on the Z-chromosome and the movement of female-biased genes from the Z-chromosome can be related to data on the abundance of sex-specific genes on the sex chromosomes of female heterogametic organisms. Kaiser and Ellegren (2006) and Storchová and Divina (2006) reported an excess of male-specific (brain) and a deficit of female-specific (brain, ovary) genes on the chicken Z-chromosome. These studies did not take into account the lack or incompleteness of dosage compensation in birds (Ellegren et al. 2007; Itoh et al. 2007) so a word of caution is needed regarding the patterns. However, genes that become up-regulated in adult ovary are under-represented on chicken Z (Mank and Ellegren 2009), and an over-representation of testis-specific genes on $\mathrm{Z}$ is seen in the female heterogametic silkworm (Arunkumar et al. 2009), in agreement with the observations reported herein.

A more detailed analysis of expression patterns in chicken ovary revealed that genes expressed in somatic granulosa cells are over-represented on the Z-chromosome, while this is not the case for genes expressed in primary oocytes (Mořkovský et al. 2009). The latter is particularly interesting in light of the recent finding that MSCI occurs in chicken oogenesis (Schoenmakers et al. 2009). This and related observations in male heterogametic systems where MSCI occurs in spermatogenesis are likely to reflect the sometimes opposing evolutionary forces operating on sex-biased genes (Vibranovski et al. 2009a). Potentially, comparison of patterns of movement for genes expressed in somatic tissue and genes expressed during meiosis could be informative for testing the hy- pothesis that MSCI is a driving force to relocation of genes between autosomes and sex chromosomes. In the present study, I found no evidence for an excess of male-biased genes expressed in somatic tissue among genes new to the Z-chromosome, nor any evidence for an excess of female-biased expressed in soma leaving the Z-chromosome. However, these observations come with a great deal of caution given that sex-biased expression in somatic tissue is much less pronounced compared with gonads. Caution should also be needed when taking the observation of a high proportion of femalebiased genes expressed in gonads among those genes leaving the Z-chromosome in support of the MSCI hypothesis. It seems reasonable to assume that most RNAs isolated from adult ovarian tissue are expressed from other than meiotic cells, e.g., granulosa cells. Perhaps more importantly, for female heterogametic systems, the MSCI hypothesis predicts that genes involved in oogenesis should move to an autosomal location to be expressed during the critical transient stage of Z-chromosome inactivation during meiosis. However, this hypothesis makes no immediate predictions for genes expressed during spermatogenesis and is thus not directly compatible with the observation of an excess of testis-specific and male-biased genes among those genes that have emerged on the avian Z-chromosome.

While male-specific genes are over-represented among those that have left the X-chromosome during mammalian evolution via retrotransposition (Emerson et al. 2004), the human and mouse $\mathrm{X}$-chromosomes have gained several multi-copy gene families that represent cancer/testis antigens (Ross et al. 2005; Mueller et al. 2008). Zhang et al. (2010) were able to solve this apparent paradox by demonstrating that the mammalian X-chromosome has recently acquired a burst of male-biased genes, an observation consistent with predictions for recessive, male-beneficial alleles under sexual antagonism. However, for older genes, there is indeed an over-representation of male-biased genes on the autosomes, which is in line with the observation of out-of-X movement. In this context, it should also be noted that the presence of multi-copy genes on the mammalian $\mathrm{X}$ resembles the situation recently reported for the chicken Z-chromosome, to which four testis-specific genes have been recruited that subsequently have been subject to massive amplification within a highly repetitive structure (Bellott et al. 2010). Hundreds of copies of these four genes exist in a region extending over $>10 \mathrm{Mb}$. Apparently, the evolution of ampliconic structures involving male-specific multi-copy genes thus seems to be a common feature of recombining sex chromosomes, irrespective of male or female heterogamety. In mammals, similar structures are seen on the male-specific Y-chromosome (Skaletsky et al. 2003; Hughes et al. 2010).

Theoretical models on the evolution of sexually antagonistic genes make different predictions for mutations depending on their dominance coefficients (Rice 1984). For recessive mutations, the scenarios described above do not hold because X-linked/Z-linked genes beneficial to the homogametic sex, but detrimental to the heterogametic sex, will be directly selected against in the latter and not selected for in the former until they reach high enough frequency to appear in homozygote form (Ellegren and Parsch 2007). Some of the deviant observations on the overall abundance of sexbiased genes in recombining sex chromosomes, an excess of early spermatogenesis genes on the mouse $X$ (Khil et al. 2004), and an excess of female-biased genes in somatic ovary cells on the chicken $\mathrm{Z}$ (Mořkovský et al. 2009) follow the expectations from recessive mutations if sexual antagonism is translated into sex-biased expression. However, most observations from both male and female heterogametic systems on the emergence and movement of sexbiased genes, if they are considered to represent sexually antago- 
nistic genes, are consistent with a situation in which mutations in sexually antagonistic genes are at least partly dominant.

To conclude, I find evidence for an over-representation of male-biased genes among those that have emerged on the Z-chromosome during avian evolution and a tendency for over-representation of female-biased genes among those that have left the Z-chromosome for an autosomal location. Overall, these data support a model of adaptive genome organization driven by natural selection. This may either be explained by a population genetic model based on sexual antagonism or, maybe less likely, be related to meiotic sex chromosome inactivation. A general remark is also that by contrasting observations on sex chromosome evolution in male and female heterogametic systems, it is possible to make independent tests of theoretical predictions on evolutionary patterns related to sex linkage of sex-specific genes.

\section{Methods}

Data on gains and losses of genes from the chicken Z-chromosome were obtained from Bellott et al. (2010). I used the interpretations made by Bellott et al. for the origin of new genes on the Z-chromosome according to the following: (1) new, bird-specific genes (no mammalian ortholog), (2) genes that have moved to the avian sex chromosomes (human ortholog located in regions of the human genome that are not syntenic with the chicken Z-chromosome), (3) duplication of autosomal bird gene with the new copy inserted to the Z-chromosome, and (4) gene annotation lacking. The genes correspond to categories (B i) and (B iii a) of Bellott et al. (2010). Gene losses from the chicken Z-chromosomes were defined as genes from those human genomic regions homologous with the chicken Z-chromosome (human autosomes 5, 9, and 18), which are syntenic with neighbors in outgroups but are autosomal in chicken. These genes correspond to category ( $\mathrm{C}$ iii b) of Bellott et al. (2010) and are interpreted to have been relocated from $\mathrm{Z}$ to an autosome during avian evolution.

Gene expression levels in adult testis and ovary of chicken were from Gene Expression Omnibus (GEO) (http://www.ncbi.nlm. nih.gov/geo/) series GSE14013 and are based on microarray hybridizations using Affymetrix GeneChip 32K transcript arrays (GEO platform GPL3213). The expression data were derived from hybridizations of two pools (four individuals in each pool) of testis samples and two pools of ovary samples from 17-wk-old chicken. Quality assessment of microarray data was done using probe level robust linear model fitting provided by the affyPLM package. The procedures for data processing are described in Moŕkovský et al. (2010) and involved using gcRMA from BioConductor with quantile normalization (http://www.bioconductor.org/) for calculation of expression levels. A gene was considered as expressed if at least one of the pools revealed a significant hybridization signal and as not expressed if none of the pools did. I defined gonad genes as male-specific if they were expressed in testis but not in ovary, and as female-specific if expressed in ovary but not in testis. For the analysis of sex-biased expression, I used the mean expression value for the two pools of each sex. I defined Z-linked genes as having malebiased expression if the male-to-female expression ratio exceeded 2.5 and as having female-biased expression if the ratio was lower than 0.75 . These criteria were set based on the realization that dosage compensation of the avian Z-chromosome is incomplete and that the mean male-to-female ratio of Z-linked genes is about 1.5 (Ellegren et al. 2007; Itoh et al. 2007).

Gene expression data from somatic tissue (heart and brain of 18-d-old embryos) were obtained from Ellegren et al. (2007) and are available under the GEO accession number GSE8693. These data were also gathered using the Affymetrix GeneChip 32K transcript array, with similar protocols and procedures as for the gonadal data. I defined sex-biased expression of Z-linked genes in the same way as above. For autosomal genes, I followed the definition of sex-biased gene expression of Ellegren et al. (2007) as genes being expressed at a significantly higher level in one sex than the other (using a Bayesian moderated t-statistic with Benjamini and Hochberg false discovery rate correction).

It has recently been suggested that analyses of microarray data can lead to false conclusions on sex chromosome dosage compensation since the relationship between hybridization intensity and transcript abundance is not linear over a wide range of transcript levels (Xiong et al. 2010). This will require further investigation using alternative techniques for measuring transcript levels, notably RNA-seq and will ultimately need large-scale proteomic data. In the case of the inference of incomplete dosage compensation in birds based on microarray data, this has recently been confirmed in an independent study using RNA-seq (Wolf and Bryk 2011).

\section{Acknowledgments}

This research was supported by the Swedish Research Council, a European Research Council Advanced Investigator Grant, and a Knut and Alice Wallenberg Foundation Wallenberg Scholar award. I thank Libor Mořkovský for details about microarray data and several reviewers for comments on earlier versions of this manuscript.

\section{References}

Arunkumar KP, Mita K, Nagaraju J. 2009. The silkworm Z chromosome is enriched in testis-specific genes. Genetics 182: 493-501.

Bellott DW, Skaletsky H, Pyntikova T, Mardis ER, Graves T, Kremitzki C, Brown LG, Rozen S, Warren WC, Wilson RK, et al. 2010. Convergent evolution of chicken $\mathrm{Z}$ and human $\mathrm{X}$ chromosomes by expansion and gene acquisition. Nature 466: 612-616.

Betran E, Thornton K, Long M. 2002. Retroposed new genes out of the X in Drosophila. Genome Res 12: 1854-1859.

Bradley J, Baltus A, Skaletsky H, Royce-Tolland M, Dewar K, Page DC. 2004. An X-to-autosome retrogene is required for spermatogenesis in mice. Nat Genet 36: 872-876.

Charlesworth B, Coyne JA, Barton NH. 1987. The relative rates of evolution of sex chromosomes and autosomes. Am Nat 130: 113146.

Dalloul RA, Long JA, Zimin AV, Aslam L, Beal K, Blomberg LA, Bouffard P Burt DW, Crasta O, Crooijmans RP, et al. 2010. Multi-platform nextgeneration sequencing of the domestic turkey (Meleagris gallopavo): Genome assembly and analysis. PLoS Biol 8: e1000475. doi: 10.1371/ journal.pbio. 1000475 .

Ellegren H. 2000. Evolution of the avian sex chromosomes and their role in sex determination. Trends Ecol Evol 15: 188-192.

Ellegren H, Parsch J. 2007. The evolution of sex-biased genes and sex-biased gene expression. Nat Rev Genet 8: 689-698.

Ellegren H, Hultin-Rosenberg L, Brunström B, Dencker L, Kultima K, Scholz B. 2007. Faced with inequality: chicken do not have a general dosage compensation of sex-linked genes. BMC Biol 5: 40. doi: 10.1186/17417007-5-40.

Emerson JJ, Kaessmann H, Betran E, Long M. 2004. Extensive gene traffic on the mammalian X chromosome. Science 303: 537-540.

Fridolfsson A-K, Cheng H, Copeland NG, Jenkins NA, Liu HC, Raudsepp T, Woodage T, Chowdhary B, Halverson J, Ellegren H. 1998. Evolution of the avian sex chromosomes from an ancestral pair of autosomes. Proc Natl Acad Sci 95: 8147-8152.

Hense W, Baines JF, Parsch J. 2007. X chromosome inactivation during Drosophila spermatogenesis. PLoS Biol 5: e273. doi: 10.1371/ journal.pbio.0050273.

Hughes JF, Skaletsky H, Pyntikova T, Graves TA, van Daalen SK, Minx PJ, Fulton RS, McGrath SD, Locke DP, Friedman C, et al. 2010. Chimpanzee and human Y chromosomes are remarkably divergent in structure and gene content. Nature 463: 536-539.

Hurst LD, Pal C, Lercher MJ. 2004. The evolutionary dynamics of eukaryotic gene order. Nat Rev Genet 5: 299-310.

International Chicken Genome Sequencing Consortium. 2004. Sequence and comparative analysis of the chicken genome provide unique perspectives on vertebrate evolution. Nature 432: 695-716. 
Itoh Y, Melamed E, Yang X, Kampf K, Wang S, Yehya N, Van Nas A, Replogle $\mathrm{K}$, Band MR, Clayton DF, et al. 2007. Dosage compensation is less effective in birds than in mammals. J Biol 6: 2 . doi: 10.1186/jbiol53.

Kaiser VB, Ellegren H. 2006. Nonrandom distribution of genes with sexbiased expression in the chicken genome. Evolution 60: 1945-1951.

Khil PP, Smirnova NA, Romanienko PJ, Camerini-Otero RD. 2004. The mouse $\mathrm{X}$ chromosome is enriched for sex-biased genes not subject to selection by meiotic sex chromosome inactivation. Nat Genet 36: 642-646.

Mank JE, Ellegren H. 2009. Sex-linkage of sexually antagonistic genes is predicted by female, but not male, effects in birds. Evolution 63: 14641472.

Meisel RP, Han MV, Hahn MW. 2009. A complex suite of forces drives gene traffic from Drosophila X chromosomes. Genome Biol Evol 1: 176188.

Mita K, Kasahara M, Sasaki S, Nagayasu Y, Yamada T, Kanamori H, Namiki N, Kitagawa M, Yamashita H, Yasukochi Y, et al. 2004. The genome sequence of silkworm, Bombyx mori. DNA Res 11: 27-35.

Mořkovský L, Storchová R, Plachý J, Ivánek R, Divina P, Hejnar J. 2010. The chicken $\mathrm{Z}$ chromosome is enriched for genes with preferential expression in ovarian somatic cells. J Mol Evol 70: 129-136.

Moyle LC, Muir CD, Han MV, Hahn MW. 2010. The contribution of gene movement to the "two rules of speciation." Evolution 64: 1541-1557.

Mueller JL, Mahadevaiah SK, Park PJ, Warburton PE, Page DC, Turner JM. 2008. The mouse $\mathrm{X}$ chromosome is enriched for multicopy testis genes showing postmeiotic expression. Nat Genet 40: 794-799.

Nanda I, Shan Z, Schartl M, Burt DW, Koehler M, Nothwang H, Grützner F Paton IR, Windsor D, Dunn I, et al. 1999. 300 million years of conserved synteny between chicken $\mathrm{Z}$ and human chromosome 9 . Nat Genet 21: 258-259.

Parisi M, Nuttall R, Naiman D, Bouffard G, Malley J, Andrews J, Eastman S, Oliver B. 2003. Paucity of genes on the Drosophila X chromosome showing male-biased expression. Science 299: 697-700.

Potrzebowski L, Vinckenbosch N, Marques AC, Chalmel F, Jégou B, Kaessmann H. 2008. Chromosomal gene movements reflect the recent origin and biology of therian sex chromosomes. PLoS Biol 6: e80. doi: 10.1371/journal.pbio.0060080.

Reinke V, Smith HE, Nance J, Wang J, Van Doren C, Begley R, Jones SJ, Davis EB, Scherer S, Ward S, et al. 2000. A global profile of germline gene expression in C. elegans. Mol Cell 6: 605-616.

Rice WR. 1984. Sex chromosomes and the evolution of sexual dimorphism. Evolution 38: 735-742.

Rice WR. 1992. Sexually antagonistic genes: Experimental evidence. Science 256: $1436-1439$.

Rice WR, Chippindale AK. 2001. Intersexual ontogenetic conflict. J Evol Biol 14: $685-693$.

Rogers DW, Carr M, Pomiankowski A. 2003. Male genes: X-pelled or X-cluded? Bioessays 25: 739-741.
Ross MT, Grafham DV, Coffey AJ, Scherer S, McLay K, Muzny D, Platzer M, Howell GR, Burrows C, Bird CP, et al. 2005. The DNA sequence of the human X chromosome. Nature 434: 325-337.

Schoenmakers S, Wassenaar E, Hoogerbrugge JW, Laven JS, Grootegoed JA Baarends WM. 2009. Female meiotic sex chromosome inactivation in chicken. PLoS Genet 5: e1000466. doi: 10.1371/journal.pgen.1000466.

Skaletsky H, Kuroda-Kawaguchi T, Minx PJ, Cordum HS, Hillier L, Brown LG, Repping S, Pyntikova T, Ali J, Bieri T, et al. 2003. The male-specific region of the human $\mathrm{Y}$ chromosome is a mosaic of discrete sequence classes. Nature 423: 825-837.

Storchová R, Divina P. 2006. Nonrandom representation of sex-biased genes on chicken Z chromosome. J Mol Evol 63: 676-681.

Sturgill D, Zhang Y, Parisi M, Oliver B. 2007. Demasculinization of X chromosomes in the Drosophila genus. Nature 450: 238-241.

van Doorn GS. 2009. Intralocus sexual conflict. Ann NY Acad Sci 1168: $52-71$.

Vibranovski MD, Lopes HF, Karr TL, Long M. 2009a. Stage-specific expression profiling of Drosophila spermatogenesis suggests that meiotic sex chromosome inactivation drives genomic relocation of testisexpressed genes. PLoS Genet 5: e1000731. doi: 10.1371/ journal.pgen.1000731.

Vibranovski MD, Zhang Y, Long M. 2009b. General gene movement off the $\mathrm{X}$ chromosome in the Drosophila genus. Genome Res 19: 897-903.

Vicoso B, Charlesworth B. 2009. The deficit of male-biased genes on the $D$. melanogaster $\mathrm{X}$ chromosome is expression-dependent: A consequence of dosage compensation? J Mol Evol 68: 576-583.

Warren WC, Clayton DF, Ellegren H, Arnold AP, Hillier LW, Künstner A, Searle S, White S, Vilella AJ, Fairley S, et al. 2010. The genome of a songbird. Nature 464: 757-762.

Wolf J, Bryk J. 2011. General lack of global dosage compensation in ZZ/ZW systems? Broadening the perspective with RNA-seq. BMC Genomics 12: 91. doi: 10.1186/1471-2164-12-91.

Wu C-I, Yujun Xu E. 2003. Sexual antagonism and X inactivation-the SAXI hypothesis. Trends Genet 19: 243-247.

Xia Q, Zhou Z, Lu C, Cheng D, Dai F, Li B, Zhao P, Zha X, Cheng T, Chai C; Biology Analysis Group, et al. 2004. A draft sequence for the genome of the domesticated silkworm (Bombyx mori). Science 306: 1937-1940.

Xiong Y, Chen X, Chen Z, Wang X, Shi S, Wang X, Zhang J, He X. 2010. RNA sequencing shows no dosage compensation of the active $X$ chromosome. Nat Genet 42: 1043-1047.

Zhang YE, Vibranovski MD, Landback P, Marais GAB, Long M. 2010. Chromosomal redistribution of male-biased genes in mammalian evolution with two bursts of gene gain on the X chromosome. PLoS Biol 8: e1000494. doi: 10.1371/journal.pbio.1000494.

Received December 9, 2010; accepted in revised form August 16, 2011. 


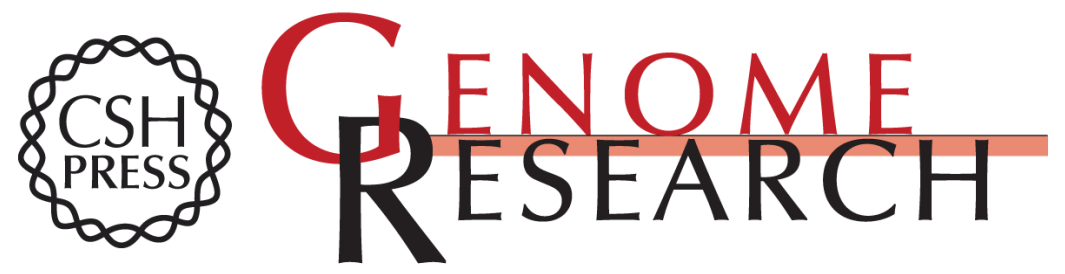

\section{Emergence of male-biased genes on the chicken Z-chromosome: Sex-chromosome contrasts between male and female heterogametic systems}

Hans Ellegren

Genome Res. 2011 21: 2082-2086 originally published online August 25, 2011

Access the most recent version at doi:10.1101/gr.119065.110

Supplemental
Material http://genome.cshlp.org/content/suppl/2011/08/29/gr.119065.110.DC1

References This article cites 47 articles, 7 of which can be accessed free at:

http://genome.cshlp.org/content/21/12/2082.full.html\#ref-list-1

\section{License}

Email Alerting

Receive free email alerts when new articles cite this article - sign up in the box at the Service top right corner of the article or click here.

\section{Affordable, Accurate Sequencing.}

